\title{
Management of Thin Gingival Biotype with Hard and Soft Tissue Augmentation Post Orthodontic Treatment: A Case Report
}

\author{
Nima Ebrahimi' ${ }^{1}$, Julio E Obando ${ }^{2}$, Michael L. Schmerman², Saba M. Khan ${ }^{2}$, Nadia I. Kawar ${ }^{2, *}$ \\ ${ }^{1}$ University of California, USA \\ ${ }^{2}$ Department of Periodontics, College of Dentistry, University of Illinois, USA
}

Copyright (C) 2015 Horizon Research Publishing All rights reserved.

\begin{abstract}
Background: In the United States, orthodontic therapy has become a common phase in people's lives. Patients seek treatment for esthetic and functional reasons. As with most treatment modalities, it has its own risks and benefits. Anatomic variations can predispose certain patients to problems. Presenting with a thin gingival tissue biotype, where arch expansion is needed, can predispose the patients for mucogingival defects and bony dehiscences. The purpose of this case report is to present a surgical approach to augment both hard and soft tissue, post orthodontic treatment to manage the resulting dehiscence and prevent potential future recession. Methods: An eighteen year old patient presented to the University of Illinois, College of Dentistry six years after completion of active orthodontics. The lower anterior segment was characterized by a thin gingival tissue biotype, prominent roots and a minimal zone of keratinized tissue. A surgical procedure was performed by placing an allogenic bone graft with subepithelial connective tissue graft and enamel matrix protein. Results: Surgical procedure augmenting hard and soft tissue was completed with uneventful post-operative healing. The goals of increased tissue thickness and prevention of recession were accomplished through this technique and the results were stable after a one year follow up.
\end{abstract}

Keywords Recession, Post-orthodontic, Hard and Soft Tissue Augmentation

\section{Introduction}

Orthodontic Therapy has become a common phase of many dental patients' life at least in the US. Patients seek orthodontic treatment for many reasons, not only esthetics, but also for function and a better overall oral health [1]. According to an NHANES III survey, the Index of Treatment Need to the reveals that $57 \%$ to $59 \%$ of each racial/ethnic group has at least some degree of orthodontic treatment need
[2]. As with most treatment and preventive modalities, orthodontic treatment is associated with potential risks and side effects. Patients present with various facial and skeletal characteristics and no orthodontal approach can fit all patients. Those anatomical variations can predispose the patients to certain risks. Thin gingival tissue biotype can predispose patients to loose supporting alveolar bone hence compromising esthetics and function. Multiple studies have associated periodontal and mucogingival defects with orthodontic treatment [3]. A retrospective study of orthodontically treated adults, found $5 \%$ prevalence of mucogingival defects [4]. A narrow band of gingiva is capable of withstanding the stress caused by orthodontic forces [5]. A study performed on adult monkeys revealed that irrespective of the soft tissue dimensions, provided that the tooth is moved within the envelope of the alveolar process, the risk of harmful effects on marginal soft tissue is minimal [6]. However, it is possible for teeth to be moved outside their alveolar housing to fulfill orthodontic therapy goals. The probability of recession during tooth movement in thin biotype is high to justify gingival augmentation when the dimension of gingiva is inadequate. In addition, cases in which there will be a facial tooth movement outside of the alveolar process need to be considered for a gingival augmentation procedure [7]. These risks should be carefully assessed prior to initiation of orthodontic therapy.

There are four main categories describing the mucogingival problems, which occur with orthodontic therapy: Labially prominent teeth, rotated teeth with labial prominence, anticipated labial movement or lingual tipping (i.e. Angle Class III correction), or distal movement of teeth with thin periodontium into an area of the alveolar ridge with narrow width [8].

An alveolar dehiscence is described as a defect of the crestal bone margin exposing the root surface [9]. Controversy exists regarding the association between lower incisor advancement and the incidence of bony dehiscences in susceptible individuals [10]. Gingival thickness had greater relevance to gingival recession when comparing 
gingival thickness to facial inclination of lower incisors [11]. A moderate association has been shown between the clinical thickness of the labial gingival and the underlying bone [12]. Thin gingival biotype, visual plaque, and inflammation are useful predictors of gingival recession [10]. Periodontal biotype is significantly related to labial plate thickness, alveolar crest position, keratinized tissue width, gingival architecture, and probe visibility but unrelated to facial recession [13]

Proper diagnosis and treatment planning is of utmost importance in minimizing the iatrogenic effects. The gingival biotype of the patient must be taken into consideration when deciding on orthodontic treatment mechanics. There are two major biotypes; a thin-scalloped and thick-flat [14]. Gingival biotypes can be categorized into three groups: flat, scalloped, and pronounced scalloped [15] . The distance from CEJ to the direct facial aspect of crestal bone normally ranges from $0.5 \mathrm{~mm}$ to $1.9 \mathrm{~mm}$ and the gingival margin is on enamel $[16,17]$. In scalloped and pronounced scalloped biotypes this distance is $2.8 \mathrm{~mm}$ and $4.1 \mathrm{~mm}$ on average respectively which results in the gingival margin being right at the CEJ or on the cementum [15].

Numerous studies have shown that a thin gingival biotype is associated with thin underlying labial plate [13]. It has been reported that thin biotypes (scalloped or pronounced scalloped) are more associated with gingival and periodontal diseases [18].

Advancement of lower anterior teeth during orthodontic treatment is done in order to reduce the overjet or increase the arch length to relieve crowding. This movement can become even more pronounced in non-extraction treatments. The increased risk of plaque accumulation throughout the course of fixed orthodontic therapy also contributes to the periodontal problems seen post orthodontic treatment [3].

Post orthodontic hard and soft tissue defects present a challenge to the treating clinician. In many cases, the connective tissue is firmly attached to exposed root surfaces and upon surgical entry these attachments are severed causing an exacerbation of the mucogingival problem [9]. Guided bone regeneration and subepithelial connective tissue grafting have been proposed as useful in treating these clinical situations [19].

The objective of this case study was to treat areas of thin soft tissue and bony dehiscences in the anterior mandible by simultaneous bone grafting and subepithelial connective tissue grafting to enhance the quality and stability of the soft and hard tissue.

\section{Case History}

An eighteen-year-old white healthy female patient presented to the Postgraduate Department of Periodontics at the University Of Illinois College Of Dentistry, Chicago, Illinois. The patient had completed orthodontic treatment approximately six years prior to being referred for a periodontal consultation regarding the mandibular anterior region. The area exhibited prominent root surfaces and minimal zone of attached and keratinized gingiva. The patient is a non -smoker, her medical and social histories were non-contributory.

A comprehensive periodontal exam was performed on the patient. Oral hygiene was determined to be fair with an O'Leary's Plaque index of $17 \%$. Periodontal probing depths ranged from 2 to $3 \mathrm{~mm}$ with no mobility in the mandibular anterior region. The mucosa overlying the labial surfaces of the lower anterior teeth appeared to be thin. The patient's gingival tissue biotype was classified as a thin-scalloped type or thin biotype [14]. Prominent roots were observable with no gingival recession noted. A zone of attached and keratinized gingiva of $1 \mathrm{~mm}$ in height throughout the labial aspect of the anterior region was noted. Figure (1) the outlines of the roots were clearly evident and prominent concavities were seen between the roots. Radiographic examination of the area revealed no apparent interproximal bone loss.

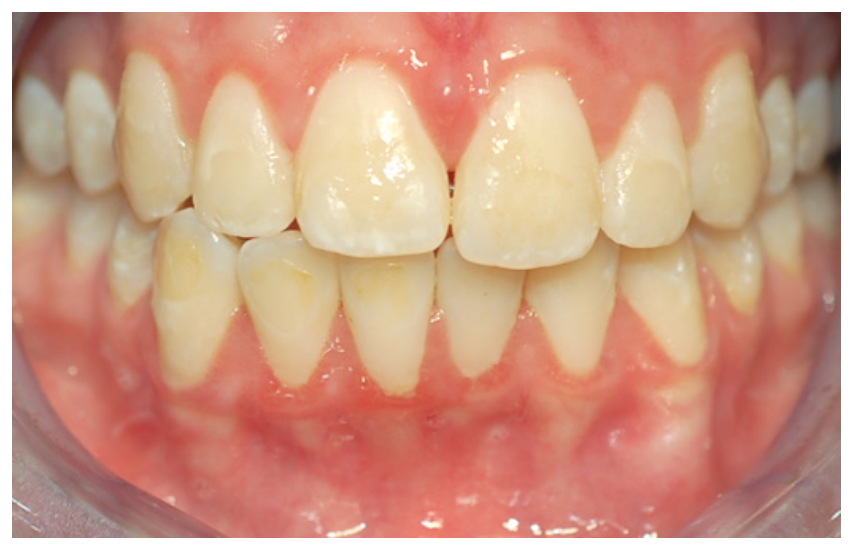

Figure (1). Pre-operative

Given the fact that the patient has a thin gingival biotype, with minimal amount of keratinized and attached gingiva in the mandibular anterior region at a young age with inconsistent plaque control, it was decided to perform a grafting procedure with the purpose of augmenting both hard and soft tissue using an autogenous subepithelial connective tissue graft procedure and an allogenic bone graft and biological material to enhance healing. This procedure would produce a thicker band of attached gingiva, increase the thickness of the labial plate which would allow for long-term stability and health of the periodontium. The findings and recommendations were explained to the patient and the patient consented to undergo the surgical procedure.

\section{Surgical Technique}

Local infiltration was used to anesthetize the surgical site using 3 cartridges (102 mg) of $2 \%$ lidocaine with 1:100,000 epinephrine. A buccal horizontal incision was made at the level of cemento-enamel junction from the distal of the lower left first premolar to the distal of the lower right first 
premolar. The papillae were kept intact. A full mucoperiosteal flap was reflected to access the defect [20]. Upon entry, dehiscences were seen on all the teeth. The lower left canine showed the largest labial dehiscence extending to the apical third of the root. The lower left lateral also exhibited a large defect extending to the apical third of the root. The defects on lower central incisors and lower right lateral incisor extended 2-4 $\mathrm{mm}$ apically from the CEJ. The lower right canine also exhibited a significant dehiscence extending to the apical third of the root. Figure (2).

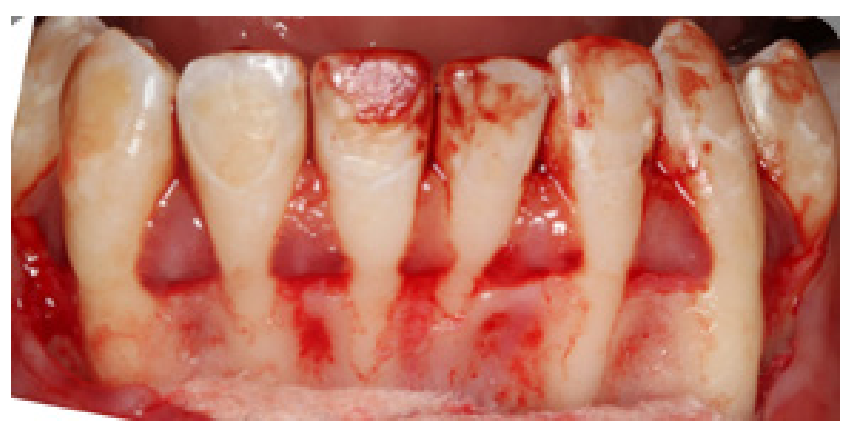

Figure (2). Full thickness flap

A $0.25 \mathrm{~mm}$ round carbide bur was used in high speed with copious irrigation for decortication of the labial cortical plate at multiple sites. Freeze dried bone allograft (FDBA) material was used for the grafting procedure. The graft material was hydrated with enamel matrix protein derivative (EMD). The EMD was also applied to the root surfaces and the bone graft material was placed over the root surfaces Figure (3)[21-26]

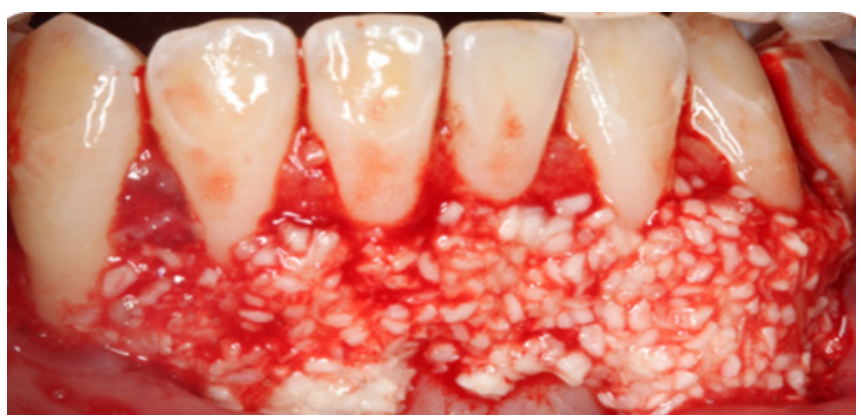

Figure (3). Grafting using FDBA and EMD

The palate was anesthetized utilizing a single cartridge (34 $\mathrm{mg}$ ) of $2 \%$ lidocaine with 1:100.000; epinephrine via greater palatine nerve block and local infiltration. Subepithelial connective tissue (SECT) graft was harvested from the palate bilaterally, Figures (4A) and (4 B). Each graft measured 4 $\mathrm{mm}$ in width and $25 \mathrm{~mm}$ in length and approximately $1.5 \mathrm{~mm}$ in thickness. Figures (5A) and (5B). Adequate hemostasis was achieved at the donor site and the site was sutured using resorbable sutures (4-0 chromic gut). The SECT grafts were positioned over the bone graft and secured in place using 5-0 chromic gut in continuous sling sutures. Figure (6) The flap was repositioned at the level of the cemento-enamel junction and single interrupted Vicryl sutures were placed. Figure (7).

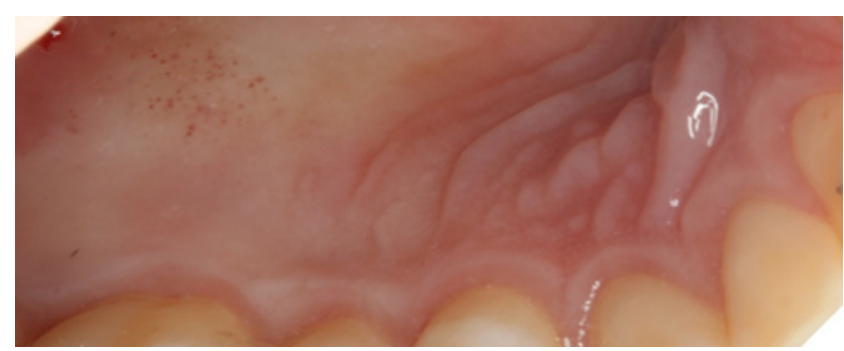

Figure (4A). Connective tissue donor site

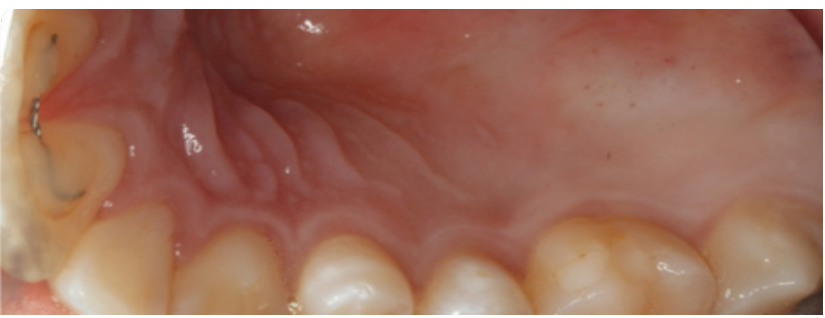

Figure (4B). Connective tissue donor site

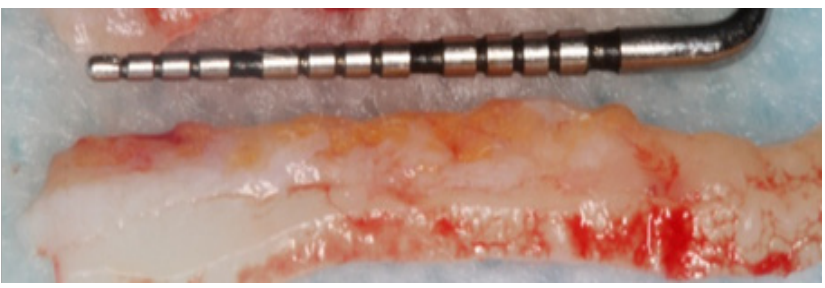

Figures (5A). Subepithelial connective tissue graft

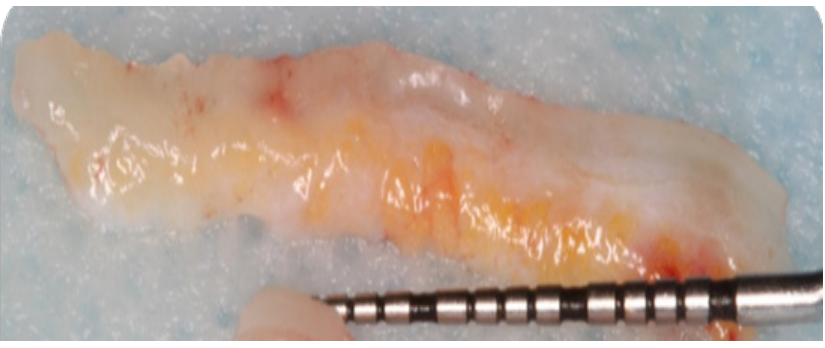

Figure (5B). Subepithelial connective tissue graft

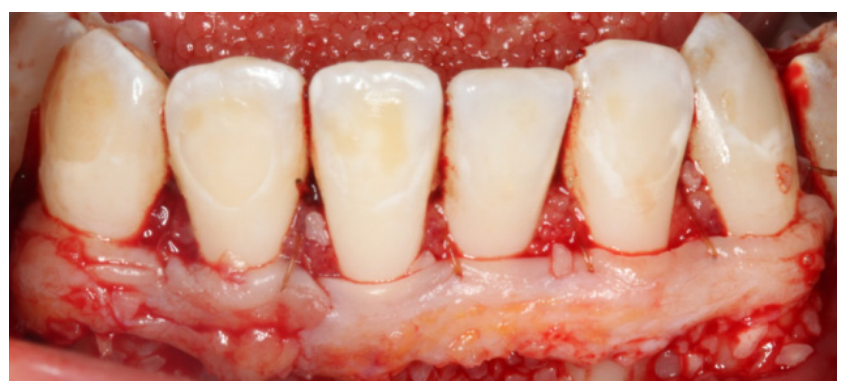

Figure (6). SECT sutured at recipient site 


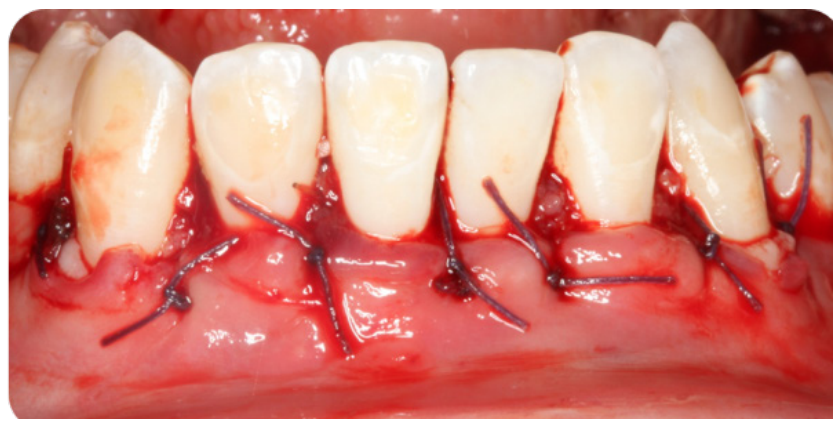

Figure (7). Flap repositioned

Periodontal dressing (Coe-Pak) was placed over the flap interlocking in the interproximal areas [27, 28]. The patient was prescribed Amoxicillin 500mg (tid for seven days), Ibuprofen $800 \mathrm{mg}$ (tid for seven days) and $0.12 \%$ Chlorhexidine rinse (bid for $30 \mathrm{~s}$ for 7 seven days) for post-operative use. Verbal and written postoperative instructions were given.

\section{Results}

The patient returned for a post-operative visit one week after the surgical procedure. Periodontal dressing was still in place. It was removed to assess the surgical site. Both recipient and donor sites were healing well without complications. The patient reported no discomfort. Sutures were still intact. Healing with primary intention noted on all the teeth. Concavities between the roots were no longer present. Sutures were left in place for an additional week. Figure (8)

The patient returned for two weeks post-operative visit, the thickness of the tissue at the lower anterior area was well increased compared to the pre-operative thickness. Figure (9)

The sites were healing satisfactorily and no gingival recession noted. Subsequent follow up visits at four weeks and six weeks the thickness of the soft tissue continued to be stable and no gingival recession was noted. Figures (10) (11)

The patient was followed up for a year and was receiving periodic maintenances, the surgical area was observed and the results were stable.

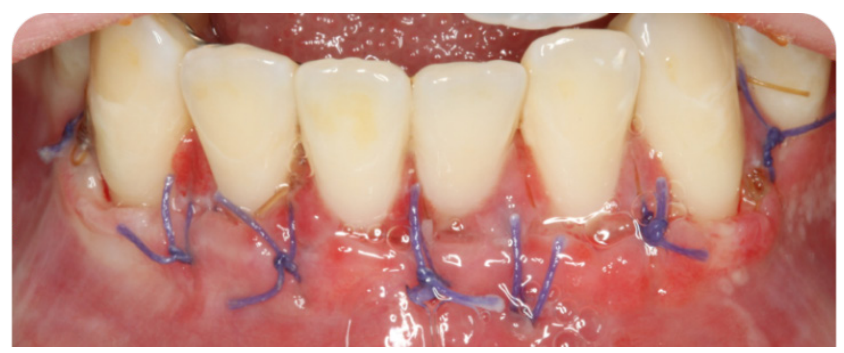

Figure (8). One week post op

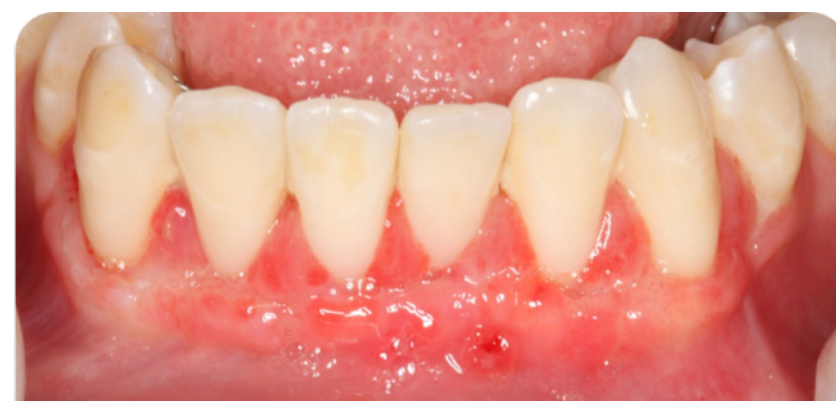

Figure (9). Two weeks post op

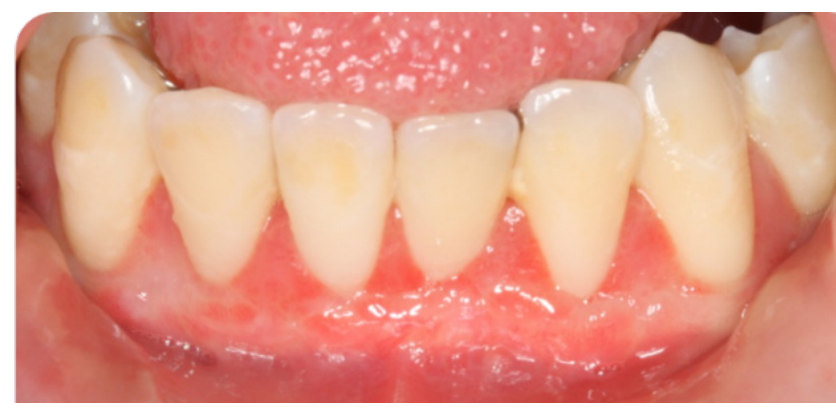

Figure (10). Four weeks post op

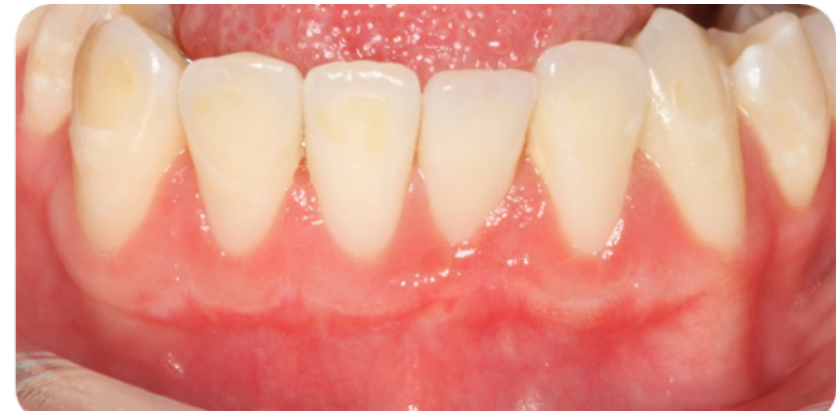

Figure (11). Six months post

\section{Discussion}

Orthodontic tooth movement may result in loss of connective tissue attachment and dehiscence when the tooth is moved out of the envelope of the alveolar process [6]. Maynard and Ochsenbein suggest that teeth that show minimal amount of keratinized gingiva pre-orthodontically are prone to more mucogingival problems following tooth movement and hence the mucogingival problems should be addressed prior to initiation of orthodontics. In addition, lingual movement of labially positioned lower incisors does not increase the width of keratinized tissue on the labial aspect of these teeth [29]. In the majority of the patients who exhibit gingival recession, the alveolar plates of bone are either thin or absent [8]. It may be concluded that soft and hard tissue defects occur simultaneously. Several considerations must be factored in when treating thin gingival tissue. The soft tissue augmentation of areas with thin gingival tissue is often accomplished by subepthelial connective tissue grafting. The hard tissue defects including 
dehiscence and fenestrations could potentially and arguably be augmented using bone graft materials.

A case series of a patient presenting a similar problem in the lower anterior region post orthodontic treatment had been published previously [19]. Two separate surgical procedures were performed to treat the case. As a first stage surgery, a bone grafting procedure using DFDBA was performed. A second stage surgery performed six months later to perform the subepithelial connective tissue grafting.

The present report represents a simultaneous augmentation of both soft and hard tissue using bone grafting material as well as connective tissue grafting procedure in a single surgical entry. Enamel matrix derivative has been used to improve the soft tissue healing and clinical attachment level in surgical root coverage procedures [30, 31]. In this clinical situation, the use of EMD might have enhanced the healing process post surgically.

No surgical re-entry was performed in this case so it is not possible to assess any regeneration and the amount of bone formation in the area without surgical re-entry or the use of Cone Beam Computed Tomography Scan (CBCT) technology. Clinically, the filling of interradicular concavities and the presence of thick connective tissue can be considered as markers of success in this particular situation, given the duration of the follow up in this patient. This patient was followed up for a year after this procedure and the results were stable. Further long-term studies are needed to support clinical and histological evidence of hard and soft tissue regeneration using the combination Enamel matrix protein derivative along with bone allograft and autogenous connective tissue graft.

It would have been optimum if a thorough assessment was performed prior to starting tooth movement orthodontically, and the need of soft and hard tissue augmentation is realized and planned before moving the teeth outside the alveolar housing. Chaturvedi R. et al presented a case of Miller's Class III gingival recession that developed in relation to the patient's lower right central incisor following orthodontic therapy [32]. In another case report, an 11-year-old girl who presented with thin gingival and minimal attached tissues in the mandibular anterior sextant. As an alternative to free autogenous grafting, an acellular dermal matrix allograft was used to augment these areas prior to orthodontic treatment, negating the requirement for a second palatal surgical procedure, the results were judged to be successful in terms of increasing the band of attached tissue, color match, ease of the procedure, and rapid recovery of the patient [33]. Another approach would have been to use an alternative orthodontic approach. The Surgically facilitated orthodontal treatment would have been another approach to this case [34, 35] the SFOT approach addresses hard tissue deficiencies and orthodontic treatment beside the other benefits of faster treatment). Although the current report is limited to a single case and the documented follow up period is relatively short, this surgical approach utilized may be used as an alternative treatment option for future cases. Given the fact that each surgery has its own risks and side effects, including further loss in the thickness of the labial plate [36, 37] a single surgical entry could be superior to conventional multi-stage surgeries especially in terms of morbidity, surgical time and cost. Further long term randomized controlled trials will shed more light on the success of this approach.

\section{Conclusions}

It is crucial to assess the amount of hard and soft tissue in comparison to teeth size and the anticipated orthodontic movement prior to starting orthodontic treatment. In patient with thin gingival biotype, labial movement of teeth outside the alveolar housing will predispose the patient for future loss of supporting labial alveolar bone and so recession compromising esthetics and function. Treating a patient after the fact that labial dehicenses has already occurred can be challenging. As single stage soft and hard tissue augmentation using biological material can be performed post-orthodontic treatment to prevent future recession.

\section{REFERENCES}

[1] de Souza, R.A., et al., Expectations of orthodontic treatment in adults: the conduct in orthodontist/patient relationship. Dental Press J Orthod, 2013. 18(2): p. 88-94.

[2] Proffit, W.R., H.W. Fields, Jr., and L.J. Moray, Prevalence of malocclusion and orthodontic treatment need in the United States: estimates from the NHANES III survey. Int J Adult Orthodon Orthognath Surg, 1998. 13(2): p. 97-106.

[3] Dannan, A., An update on periodontic-orthodontic interrelationships. J Indian Soc Periodontol, 2010. 14(1): p. 66-71.

[4] Trossello, V.K. and A.A. Gianelly, Orthodontic treatment and periodontal status. J Periodontol, 1979. 50(12): p. 665-71.

[5] Dorfman, H.S., Mucogingival changes resulting from mandibular incisor tooth movement. Am J Orthod, 1978. 74(3): p. 286-97.

[6] Wennstrom, J.L., et al., Some periodontal tissue reactions to orthodontic tooth movement in monkeys. J Clin Periodontol, 1987. 14(3): p. 121-9.

[7] Kim, D.M. and R. Neiva, Periodontal soft tissue non-root coverage procedures: a systematic review from the AAP regeneration workshop. J Periodontol, 2015. 86(2 Suppl): p. S56-72.

[8] Maynard, J.G., The rationale for mucogingival therapy in the child and adolescent. Int J Periodontics Restorative Dent, 1987. 7(1): p. 36-51.

[9] Watson, W.G., Expansion and fenestration or dehiscence. Am J Orthod, 1980. 77(3): p. 330-2.

[10] Melsen, B. and D. Allais, Factors of importance for the development of dehiscences during labial movement of mandibular incisors: a retrospective study of adult 
orthodontic patients. Am J Orthod Dentofacial Orthop, 2005. 127(5): p. 552-61; quiz 625.

[11] Yared, K.F., E.G. Zenobio, and W. Pacheco, Periodontal status of mandibular central incisors after orthodontic proclination in adults. Am J Orthod Dentofacial Orthop, 2006. 130(1): p. 6.e1-8.

[12] Fu, J.H., et al., Tissue biotype and its relation to the underlying bone morphology. J Periodontol, 2010. 81(4): p. 569-74.

[13] Cook, D.R., et al., Relationship between clinical periodontal biotype and labial plate thickness: an in vivo study. Int J Periodontics Restorative Dent, 2011. 31(4): p. 345-54.

[14] Seibert JL, L.J., Esthetics and periodontal therapy. 2nd ed. 1989: Textbook of Clinical Periodontology.

[15] Becker, W., et al., Alveolar bone anatomic profiles as measured from dry skulls. Clinical ramifications. J Clin Periodontol, 1997. 24(10): p. 727-31.

[16] Kallestal, C. and L. Matsson, Criteria for assessment of interproximal bone loss on bite-wing radiographs in adolescents. J Clin Periodontol, 1989. 16(5): p. 300-4.

[17] Hausmann, E., K. Allen, and V. Clerehugh, What alveolar crest level on a bite-wing radiograph represents bone loss? J Periodontol, 1991. 62(9): p. 570-2.

[18] Claffey, N. and D. Shanley, Relationship of gingival thickness and bleeding to loss of probing attachment in shallow sites following nonsurgical periodontal therapy. J Clin Periodontol, 1986. 13(7): p. 654-7.

[19] Bonacci, F.J., Hard and soft tissue augmentation in a postorthodontic patient: a case report. Int $\mathrm{J}$ Periodontics Restorative Dent, 2011. 31(1): p. 19-27.

[20] Langer, B. and L. Langer, Subepithelial connective tissue graft technique for root coverage. J Periodontol, 1985. 56(12): p. 715-20.

[21] Boyan, B.D., et al., Porcine fetal enamel matrix derivative enhances bone formation induced by demineralized freeze dried bone allograft in vivo. J Periodontol, 2000. 71(8): p. 1278-86.

[22] Al-Hezaimi, K., et al., The effect of enamel matrix protein on gingival tissue thickness in vivo. Odontology, 2012. 100(1): p. 61-6.

[23] Sculean, A., et al., Emdogain in regenerative periodontal therapy. A review of the literature. Fogorv Sz, 2007. 100(5): p. 220-32, 211-9.

[24] Koop, R., J. Merheb, and M. Quirynen, Periodontal regeneration with enamel matrix derivative in reconstructive periodontal therapy: a systematic review. J Periodontol, 2012. 83(6): p. 707-20.

[25] Trabulsi, M., et al., Effect of enamel matrix derivative on collagen guided tissue regeneration-based root coverage procedure. J Periodontol, 2004. 75(11): p. 1446-57.

[26] Aspriello, S.D., Effects of enamel matrix derivative on vascular endothelial growth factor expression and microvessel density in gingival tissues of periodontal pocket: a comparative study. J Periodontol, 2011. 82(4): p. 606-12.

[27] Baghani, Z. and M. Kadkhodazadeh, Periodontal dressing: a review article. J Dent Res Dent Clin Dent Prospects, 2013. 7(4): p. 183-91.

[28] Sachs, H.A., et al., Current status of periodontal dressings. J Periodontol, 1984. 55(12): p. 689-96.

[29] Maynard, J.G., Jr. and C. Ochsenbein, Mucogingival problems, prevalence and therapy in children. J Periodontol, 1975. 46(9): p. 543-52.

[30] Chambrone, L., et al., Evidence-based periodontal plastic surgery. II. An individual data meta-analysis for evaluating factors in achieving complete root coverage. J Periodontol, 2012. 83(4): p. 477-90.

[31] Henriques, P.S., et al., Application of subepithelial connective tissue graft with or without enamel matrix derivative for root coverage: a split-mouth randomized study. J Oral Sci, 2010. 52(3): p. 463-71.

[32] Chaturvedi R et al, Mucogingival considerations following orthodontic therapy: a case report.Compend Contin Educ Dent. 2011 Oct;32(8):36, 38-41.

[33] Fowler EB1et al, Mil Med. Use of acellular dermal matrix allograft for management of inadequate attached gingiva in a young patient. Mil Med. 2003 Mar;168(3):261-5.

[34] K, S., et al., Wilckodontics - a novel synergy in time to save time. J Clin Diagn Res, 2014. 8(1): p. 322-5.

[35] Roblee, R.D., S.L. Bolding, and J.M. Landers, Surgically facilitated orthodontic therapy: a new tool for optimal interdisciplinary results. Compend Contin Educ Dent, 2009. 30(5): p. 264-75; quiz 276, 278.

[36] Wood, D.L., et al., Alveolar crest reduction following full and partial thickness flaps. J Periodontol, 1972. 43(3): p. $141-4$.

[37] Fickl, S., et al., Bone loss after full-thickness and partial-thickness flap elevation. J Clin Periodontol, 2011. 38(2): p. 157-62. 